\title{
Erratum to: Algebras of Ehresmann semigroups and categories
}

\author{
Itamar Stein 1
}

Received: 4 May 2017 / Accepted: 30 July 2017 / Published online: 21 August 2017

(C) Springer Science+Business Media, LLC 2017

\section{Erratum to: Semigroup Forum DOI 10.1007/s00233-016-9838-1}

Shoufeng Wang discovered an error in the main theorem of the author's Semigroup Forum article 'Algebras of Ehresmann semigroups and categories'. Wang observed that the function we suggest as an isomorphism is not a homomorphism unless the semigroup being discussed is left restriction. In order to fix our mistake, we will add this assumption. Note that our revised result is still a generalization of earlier work of Guo and Chen, the author, and Steinberg.

\section{A correction to the main theorem of [3]}

Shoufeng Wang [6] has observed that the proof of [3, Theorem 3.4] does not hold without the additional assumption that the semigroup is left restriction. This Erratum shows how this assumption yields a valid result, and examines the consequences. Theorem 1.5, which is the revision of [3, Theorem 3.4], nevertheless generalizes [5, Theorem 4.2], [2, Theorem 4.2] and [4, Proposition 3.2].

We assume the reader is familiar with [3] and in particular with the definition of an $E$-Ehresmann semigroup; for undefined terms, the reader should consult [3].

Communicated by Victoria Gould.

The online version of the original article can be found under doi:10.1007/s00233-016-9838-1.

$凶 \quad$ Itamar Stein

Steinita@gmail.com

1 Department of Mathematics, Bar Ilan University, 52900 Ramat Gan, Israel 
We start by giving a counterexample to the original claim in [3, Theorem 3.4]. Let $S$ be an $E$-Ehresmann semigroup with $\leq=\leq_{r}$ a principally finite poset, and let $C=\mathbf{C}(S)$ be the corresponding Ehresmann category. We do not know whether $\mathbb{K} S$ is always isomorphic to $\mathbb{K} C$, but Shoufeng Wang observed that the function $\varphi: \mathbb{K} S \rightarrow \mathbb{K} C$ defined by

$$
\varphi(a)=\sum_{b \leq a} C(b)
$$

is, in general, not a homomorphism, as can be seen in the following example.

Example 1.1 Choose $S=B_{2}$, the monoid of all binary relations on the set $\{1,2\}$. As mentioned in [3, Example 4.4], $B_{2}$ is an $E$-Ehresmann semigroup where $E=$ $\{$ id, $\{(1,1)\},\{(2,2)\}, \varnothing\}$ is the set of partial identities. For every $a \in B_{2}, a^{+}\left(a^{*}\right)$ is the identity function on the domain (respectively, image) of $a$. Let $C=\mathbf{C}\left(B_{2}\right)$ be the corresponding Ehresmann category. It is easy to see that $\varphi: \mathbb{K} B_{2} \rightarrow \mathbb{K} C$ is not a homomorphism. Choose $a=\{(1,1),(1,2)\}$ and $b=\{(1,1)\}$ so $a b=\{(1,1)\}$. Note that in $B_{2}, \leq$ is domain restriction, so

$$
\varphi(b)=\varphi(a b)=\varphi(\{(1,1)\})=C(\{(1,1)\})+C(\varnothing)
$$

and

$$
\varphi(a)=C(\{(1,1),(1,2)\})+C(\varnothing) .
$$

Recall that in the category algebra the multiplication $x y$ equals 0 unless $\mathbf{r}(x)=\mathbf{d}(y)$. Hence,

$$
\varphi(a) \varphi(b)=(C(\{(1,2),(1,1)\})+C(\varnothing))(C(\{(1,1)\})+C(\varnothing))=C(\varnothing)
$$

so indeed

$$
\varphi(a b) \neq \varphi(a) \varphi(b)
$$

In order to fix this problem, we will have to add the requirement of being left (or right) restriction.

Definition 1.2 Let $S$ be a left $E$-Ehresmann semigroup. $S$ is called left restriction if

$$
a e=(a e)^{+} a
$$

for every $a \in S$ and $e \in E$. Dually, a right $E$-Ehresmann semigroup $S$ is called right restriction if

$$
e a=a(e a)^{*}
$$

for every $a \in S$ and $e \in E$. If $S$ is an $E$-Ehresmann semigroup which is both left and right restriction, then it is called a restriction semigroup. 
Example 1.3 Every inverse semigroup is a restriction semigroup. It is well known that the monoid $\mathcal{P} \mathcal{T}_{n}$ of all partial transformations on an $n$-element set is left restriction but not right restriction. However, the monoid $B_{n}$ of all binary relations on an $n$-element set is neither left nor right restriction.

Lemma 1.4 If $S$ is an E-Ehresmann semigroup which is also left restriction (right restriction), then $\leq_{l} \subseteq \leq_{r}$ (respectively, $\leq_{r} \subseteq \leq_{l}$ ).

Proof Assume $b \leq_{l} a$. By [3, Proposition 2.6], there exists an $e \in E$ such that $b=a e=(a e)^{+} a$ but $(a e)^{+} \in E$ so $b \leq_{r} a$ as well. The other case is dual.

We can now give a correct version of [3, Theorem 3.4], under the additional assumption of being left restriction.

Theorem 1.5 Let $S$ be an E-Ehresmann and left restriction semigroup and let $C=$ $\mathbf{C}(S)$. Then $\mathbb{K} S$ is isomorphic to $\mathbb{K} C$. Explicit isomorphisms $\varphi: \mathbb{K} S \rightarrow \mathbb{K} C, \psi:$ $\mathbb{K} C \rightarrow \mathbb{K} S$ are defined (on basis elements) by

$$
\varphi(a)=\sum_{b \leq a} C(b), \quad \psi(x)=\sum_{y \leq x} \mu(y, x) S(y)
$$

where $\mu$ is the Möbius function of the poset $\leq$.

Remark 1.6 Theorem 1.5 can be proved, mutatis mutandis, for $E$-Ehresmann and right restriction semigroups using $\leq_{l}$ instead of $\leq_{r}$.

Proof of Theorem 1.5 The proof that $\varphi$ and $\psi$ are bijections is identical to that in [3], as is Case 1 of the proof that $\varphi$ is a homomorphism.

For Case 2, we assume $\mathbf{r}(C(a)) \neq \mathbf{d}(C(b))$ (or equivalently, $a^{*} \neq b^{+}$). Define $\tilde{a}=a b^{+}$and $\tilde{b}=a^{*} b$. Proceeding as in [3], it remains to show that

$$
\left(\sum_{a^{\prime} \leq \tilde{a}} C\left(a^{\prime}\right)\right)\left(\sum_{b^{\prime} \leq \tilde{b}} C\left(b^{\prime}\right)\right)=\left(\sum_{a^{\prime} \leq a} C\left(a^{\prime}\right)\right)\left(\sum_{b^{\prime} \leq b} C\left(b^{\prime}\right)\right) .
$$

We set $x=C(a), \tilde{x}=C(\tilde{a}), y=C(b)$ and $\tilde{y}=C(\tilde{b})$ so (1) can be written as

$$
\left(\sum_{x^{\prime} \leq \tilde{x}} x^{\prime}\right)\left(\sum_{y^{\prime} \leq \tilde{y}} y^{\prime}\right)=\left(\sum_{x^{\prime} \leq x} x^{\prime}\right)\left(\sum_{y^{\prime} \leq y} y^{\prime}\right) .
$$

Now we need the new assumption of left restriction. First note that $\tilde{y} \leq y$ and $\tilde{x} \leq_{l} x$ since they are restriction and co-restriction, respectively. $S$ is left restriction so $\tilde{x} \leq x$ by Lemma 1.4. Hence, every element on the left-hand side of (2) appears also on the right-hand side. What is left to show is that a multiplication $x^{\prime} \cdot y^{\prime}$ on the right-hand side of (2) equals 0 unless $x^{\prime} \leq \tilde{x}$ and $y^{\prime} \leq \tilde{y}$. Take $x^{\prime} \leq x$ such that $x^{\prime} \leq \tilde{x}$ and assume that there is a $y^{\prime} \leq y$ such that $\exists x^{\prime} \cdot y^{\prime}$, that is, $\mathbf{r}\left(x^{\prime}\right)=\mathbf{d}\left(y^{\prime}\right)$. Since $y^{\prime} \leq y$ 
we have $\mathbf{r}\left(x^{\prime}\right)=\mathbf{d}\left(y^{\prime}\right) \leq \mathbf{d}(y)$ by [3, CO1]. Now, by [3, EC7] (choosing $f=\mathbf{d}(y)$ ) we have that

$$
\left(x^{\prime} \mid \mathbf{r}\left(x^{\prime}\right) \wedge \mathbf{d}(y)\right) \leq(x \mid \mathbf{r}(x) \wedge \mathbf{d}(y))
$$

but note that $(x \mid \mathbf{r}(x) \wedge \mathbf{d}(y))=\tilde{x}$ and $\mathbf{r}\left(x^{\prime}\right) \wedge \mathbf{d}(y)=\mathbf{r}\left(x^{\prime}\right)$ so we get

$$
x^{\prime}=\left(x^{\prime} \mid \mathbf{r}\left(x^{\prime}\right)\right) \leq \tilde{x}
$$

a contradiction. Similarly, take $y^{\prime} \leq y$ such that $y^{\prime} \not \tilde{y}$ and assume that there is an $x^{\prime} \leq x$ such that $\exists x^{\prime} \cdot y^{\prime}$, that is, $\mathbf{r}\left(x^{\prime}\right)=\mathbf{d}\left(y^{\prime}\right)$. Again, since $\mathbf{r}\left(x^{\prime}\right) \leq \mathbf{r}(x)$ we have that $\mathbf{d}\left(y^{\prime}\right) \leq \mathbf{r}(x)$ and clearly $\mathbf{d}\left(y^{\prime}\right) \leq \mathbf{d}(y)$, hence $\mathbf{d}\left(y^{\prime}\right) \leq \mathbf{r}(x) \wedge \mathbf{d}(y)=\mathbf{d}(\tilde{y})$. By [3, EC2], there exists a restriction $\left(\mathbf{d}\left(y^{\prime}\right) \mid \tilde{y}\right)$. But $\left(\mathbf{d}\left(y^{\prime}\right) \mid \tilde{y}\right) \leq \tilde{y} \leq y$ so by the uniqueness of restriction $\left(\mathbf{d}\left(y^{\prime}\right) \mid \tilde{y}\right)=y^{\prime}$, hence $y^{\prime} \leq \tilde{y}$, a contradiction. This finishes the proof.

\section{Consequences for the rest of [3]}

[3, Corollary 3.6] should be reformulated as:

Corollary 2.1 Let $S$ be an E-Ehresmann and left (or right) restriction semigroup such that $E$ is finite, then $\mathbb{K} S$ is a unital algebra.

[3, Example 4.4] should be deleted because our weakened theorem no longer applies. The rest of the examples in [3, Section 4] remain valid. In particular, note that the monoid $\mathcal{P} \mathcal{T}_{n}$ of [3, Example 4.3] is left restriction and that [1, 2.5.11-2.5.12] proves that every strong semilattice of monoids ([3, Example 4.5]) is a restriction semigroup.

The results of [3, Subsection 5.1] remain valid, but one can draw conclusions regarding the algebras of the Ehresmann semigroups only if they are left (or right) restriction. For instance, it can be done for $(2,1,1)$ subalgebras of $\mathcal{P} \mathcal{T}_{n}$ ([3, Example 5.10]) and finite ample semigroups (part of [3, Example 5.11]).

Likewise, the results of [3, Subsection 5.2] are now valid only under the additional assumption of left or right restriction. [3, Proposition 5.17] should be reformulated as:

Proposition 2.2 Let $S$ be a finite E-Ehresmann and left (or right) restriction semigroup whose corresponding Ehresmann category $C$ is an EI-category. Then $\mathbb{K} \operatorname{Reg}_{E}(S)$ is isomorphic to $\mathbb{K} S / \operatorname{Rad}(\mathbb{K} S)$.

The proof is as given in [3].

\section{References}

1. Cornock, C.: Restriction semigroups: structure, varieties and presentations. PhD thesis, University of York (2011)

2. Guo, X., Chen, L.: Semigroup algebras of finite ample semigroups. Proc. R. Soc. Edinb. Sect. A 142(2), 371-389 (2012) 
3. Stein, I.: Algebras of Ehresmann semigroups and categories. Semigroup Forum. doi:10.1007/s00233016-9838-1 (to appear)

4. Stein, I.: The representation theory of the monoid of all partial functions on a set and related monoids as EI-category algebras. J. Algebra 450, 549-569 (2016)

5. Steinberg, B.: Möbius functions and semigroup representation theory. J. Combin. Theory Ser. A 113(5), 866-881 (2006)

6. Wang, S.: Private Communication 